\title{
Establishment and decay of orientation-contingent aftereffects of color*
}

\author{
LORRIN A. RIGGS, KEITH D. WHITE, and PETER D. EIMAS
}

Brown University, Providence, Rhode Island 02912

\begin{abstract}
We have used a null method to measure the orientation-contingent aftereffects of color first described by McCollough. After alternately inspecting, for example, a green horizontal line grating and a magenta vertical line grating, the 0 s report that in achromatic test gratings the horizontal lines appear pinkish and the vertical lines appear greenish. We have used a special color-mixing projector to add variable amounts of green and magenta light to the test gratings until they appear matched and nearly achromatic. The colorimetric purity needed to achieve this null setting is a quantitative measure of the strength of the colored aftereffect. Following inspections of the colored patterns ranging from 15 sec to $150 \mathrm{~min}$, six Os showed aftereffects lasting from a few minutes to 7 or more days. The indices of colorimetric purity increase with inspection time and decline with time after inspection. The decay function is not quite linear either on semilog or on log-log coordinates. The rate of decay is mainly dependent on the magnitude of the effect built up during inspection. We conclude that the buildup and decay of these aftereffects show some of the time characteristics usually associated with central adaptability rather than sensory adaptation.
\end{abstract}

We have measured the time courses of establishment and decay of the perceptual aftereffect described by McCollough (1965). This effect, in common with other forms of perceptual adaptation is thought to be mediated by central rather than peripheral neural units, as evidenced by its slow rate of establishment and disappearance. The McCollough effect can also be described as a nonverbalized, nonmotor form of change in the central nervous system induced by relatively prolonged exposure to particular stimulus configurations. In this case, exposure is to an array of vertical lines of one color alternated with horizontal lines of another color. Continuance of the exposure produces a gradual shift in the chromatic appearance of patterns: in subsequent testing, achromatic lines appear to be oppositely colored with respect to lines of the same orientation used for inspection. The aftereffect colors, while never as vivid as those seen in sensory afterimages, are nevertheless sufficiently strong and reliable to permit measurement over a considerable range of times of establishment and decay.

The McCollough effect is but one form of perceptual adaptation. Other forms are the distortion or displacement of the visual field by prisms, reversal or inversion of the field by mirrors or lenses, and a number of spatial aftereffects that include figural aftereffects and aftereffects of motion, size, orientation, spatial frequency, and curvature. See

*This research was conducted in the Hunter Laboratory of Psychology at Brown University, with the support of PHS Grant EY-00744 and an NSF predoctoral fellowship to K.D.W. general discussions by Harris (1965), Rock (1966), Epstein (1967), Hochberg (1971), and Murch (1973). A beginning has also been made at the study of adaptation to auditory features underlying speech perception (Eimas, Cooper, \& Corbit, 1973). Despite the strong interest in these phenomena, however, relatively few experiments have measured the time courses of their establishment and decay. We trust that some of our quantitative findings and generalizations about the time course of the McCollough effect may be applicable to these other phenomena as well. If so, it may make possible specific comparisons on the time dimension, between perceptual adaptation and peripheral or sensory adaptation, on the one hand, and the "nonperceptual" central processes such as verbal and motor learning and forgetting, conditioning, and extinction, on the other.

\section{PROCEDURE}

\section{Equipment for Producing Pattern-Contingent Aftereffects}

The arrangement for presenting the colored patterns used for inspection was similar to that described by McCollough (1965). As shown in Fig. 1, the $O$ was $150 \mathrm{~cm}$ from a screen on which either inspection or test patterns were projected. Inspection patterns used to build up the effect in half the sessions were a green horizontal line grating alternated with a magenta vertical grating. In the other half of the sessions, the horizontal grating was magenta and the vertical grating was green. Each grating was projected from a standard $35-\mathrm{mm}^{\prime}$ slide projector through Eastman Kodak filters that are a nearly complementary pair (Stromeyer, Lange, \& Ganz, 1973). namely a Wratten No. 53 filter for green and a Wratten No. 34A filter for magenta. A $180-\mathrm{deg}$ sector disk, driven by a synchronous motor, was placed before the projectors in such a way that the red and green beams were allowed to reach the screen alternately for intervals of $5 \mathrm{sec}$ each. 
As vicwed from the Os' position, each inspection pattern was $7 \mathrm{deg} 13 \mathrm{~min}$ in height and width, with a bright-stripe luminance of approximately $5.2 \mathrm{fL}$. Each pattern consisted of black and bright stripes of equal width, and the spatial frequency was kept constant at 3.2 cycles $/ \mathrm{deg}$. The $O$ was told to fixate the center of the inspection field with both eyes.

\section{Equipment for Testing the Aftereffect Colors}

A special test apparatus, also shown in Fig. 1, was used to measure the strength of the colored aftereffect. This was built from a third $35-\mathrm{mm}$ slide projector that was used to place a square test patiern on the screen. Not shown in Fig. 1 is an additional source of light, namely a cool white fluorescent fixture that provided general ambient illumination. The pattern had four quadrants consisting of two horizontal-stripe and two vertical-stripe gratings. The horizontal stripes were covered with polarizing filters having a particular orientation, while the vertical stripes were covered by orthogonally oriented polarizing filters. In front of the projection lens. at a position corresponding to the focal plane of the tungsten tilament source, a rotatable disk was mounted so as to be centered on, and normal to, the projected beam of light. Portions of the filament image traversed three separate apertures near the center of a rotatable disk. One aperture contained a piece of green gelatine covered with a polarizing filter, another contained a piece of magenta gelatine covered with a polarizing filter of orthogonal orientation. and the third aperture was left clear. The effect of this was to supply the test pattern on the screen with a mixture of green, magenta, and white light. Furthermore, the relative amounts of green and magenta in the horizontal or vertical test patterns could be adjusted by changing the orientations of the gelatine-covered polarizing filters with respect $t$ hose covering the horizontal and vertical gratings on the test slide, e., by rotating the disk. At one extreme of rotation, the horizon' : received only green plus white light. while at the other extreme, it received only magenta plus white. Between these extremes, a position could be found in which green and magenta were mixed in any desired proportions. Meanwhile, the opposite effects were occurring in the vertical gratings, so that adding or subtracting green light to the horizontals was always acconpanied by a corresponding change in magenta light going to the verticals.

The disk had a large diameter and rotated smoothly, with the scale not visible to the $O$. The outer portion of the disk was smooth, so that the $O$ had no way of judging its position except by its effect on the appearance of the test patterns. In the absence of orientation-contingent aftereffects of color, an $O$ could find a neutral setting for the rotatable disk such that nearly equal amounts of green and magenta were present in the horizontal and vertical test patterns; they therefore appeared matched and nearly achromatic. This balance appeared as a mismatch, however. whenever colored aftereffects were present. A new null point was then found by rotating the disk so as to add more green to one set of lines and more magenta to the other. The direction and extent of rotation required to achieve the match depended on the direction and magnitude of the aftereffect. Hence, a scale of angular rotation, mounted on the disk, could be used to specify these values. Furthermore, the scale was calibrated in terms of the colorimetric purity of the light present on each of the quadrants of the test pattern. This was done by separately measuring the relative luminances of the green, magenta, and white components in each quadrant of the test fields at each scale position. ${ }^{1}$ Thus, the results of the present experiments are all expressed in terms of the changes in colorimetric purity that are needed to make a color match between quadrants of the test field, i.e., to cancel the aftereffect color that is present at the time of testing.

In choosing the above procedure, we have simplified the construction of equipment and facilitated the task of matching the test fields. We have rejected the more elaborate and less reliable method of having the $O$ make a separate colorimetric determination of each of his attereffect colors. Our null procedure, however, has been developed at the cost of the following constraints. First, the

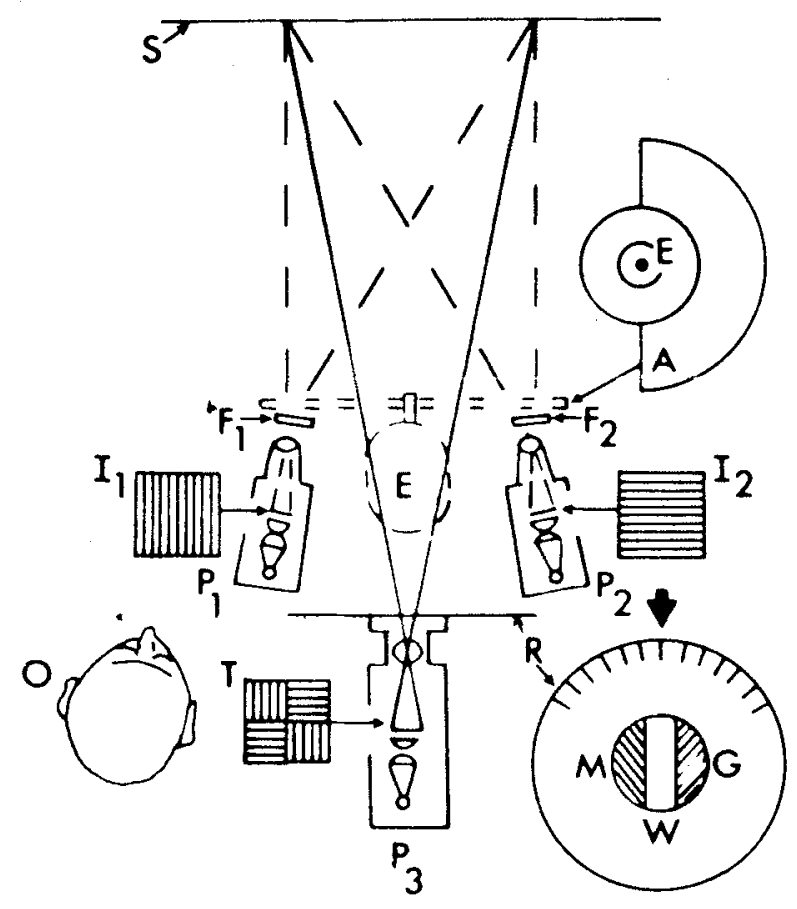

Fig. 1. Projection arrangement for inspection and testing of pattern-contingent colors (not to scale). Projectors $P_{1}$ and $P_{2}$ are turned on for the required interval to present Inspection Patterns $I_{1}$ and $I_{2}$ on the screen at $S$, with colors determined by Selective Filters $F_{1}$ and $F_{2}$. Electric motor, $E$, drives 180 -deg sector disk, $A$, to interrupt the beams alternately. After the inspection is completed, Projector $P_{3}$ is turned on and observer, $O$, rotates disk, $R$, to produce a color match betwen the horizontal and vertical quadrants of the image on the screen of test slide, $T$. These colors are regulated by the relative orientations of a pair of orthogonally polarized filters in the test slide, $T$, and a second pair of orthogonally polarized filters in the magenta $(M)$ and green $(G)$ portion of the beam from Projector $P_{3}$. A white portion $(W)$ of that beam reaches the screen through a clear aperture, to reduce the colorimetric purity of the test patterns on the screen.

test color must be exactly complementary to the aftereffect color in order that a perfect cancellation can take place. This was achieved only approximately. The green and magenta filters occupy nearly complementary positions on the CIE chromaticity diagram. But when the aftereffects were at their strongest, Os sometimes reported that they could not find any position on the test mixture in which an achromatic setting could be achieved for either the horizontal or the vertical line pattern. Second, the separate contributions of the green and magenta components of the test field must be balanced so that cancellation of the aftereffect color appearing in the horizontal stripes occurs at the same scale position as cancellation of the other aftereffect color appearing in the vertical stripes. This, too. w:as only approximatcly achieved, and some differences in this balance were found from one $\mathrm{O}$ to another. The situation was substantially remedied by making two versions of the four-quadrant test slide. Corresponding quadrants of the two were oppositely polarized so that, given a constant direction of aftereffect, the rotatable disk was set on opposite sides of the scale when using the two test slides. This is the optical (and much more comfortable) equivatent of asking the $\mathrm{O}$ to turn his head through $90 \mathrm{deg}$ to make a second set of matches. Averaging these two measures of colorimetric purity produced a satisfactory degree of stability in the individual runs, even though $O$ s sometimes differed in their absolute scale distances on the two sides of the zero point. Third, 
the filament image must be completely homogeneous in the plane of the rotatable disk. Since this was not perfectly achieved, different areas of the test pattern exhibited slight differences in color as the disk was turned. The practical solution was to instruct the Os to fixate on one particular point in a border between quadrants of the field for making their test matches and to disregard the slight variations of color that appeared in other parts of the field. Fourth, the strongest aftereffects must not exceed the limits of the color-mixing test system. The longest inspections, however, often produced such vivid colors that the Os went off the scale without attaining a proper cancellation. A partial solution was again found, namely to cover up a portion of the clear aperture in the disk so as to reduce the relative proportion of white light on the screen. This was sufficient in most cases: but those Os attaining the strongest aftereffects of color still could not begin to make settings until a partial decay of the effects had brought them down to the measurable range.

The effect of all the above limitations is not to invalidate our data, but rather to impose some limitations on the total extent of the effects that we could measure and, no doubt, to reduce somewhat the precision of settings at the high end of the scale. To climinate most of these difficulties, we have now built a new apparatus in which the inspection colors are more nearly complementary, being from Wratten filters No. 53 and No. 32. The filters in the rotatable disk are not the same as those used for inspection; Wratten filters CC $30 \mathrm{G}$ and CC20M are used because they have the right hues and colorimetric purities so that no white light needs to be added, and yet a strong enough balancing color can be produced to cancel all aftereffects. The filament image in front of the projector is now sufficiently well sampled, by a large array of small pieces of both color filters, so that the test fields appear homogeneous. The new test field contains only two sections to be matched in color; a single thin line separates the sections containing horizontal and vertical stripes.

\section{Subjects}

Three male Os (authors of this paper) and three females (an undergraduate student and two research assistants) were given varying amounts of preliminary training in making the null settings on the test pattern until they became accustomed to the procedures and gave reliable results. One male (K.W.) and one female (J.F.) showed the strongest aftereffects of color; one male (L.R.) and one female (J.C.) showed aftereffects of intermediate strength; and one male (P.E.) and one female (G.A.) showed the weakest effects.

Each $O$, on separate days, was given inspection periods having approximately equal logarithmic steps, namely $15 \mathrm{sec}, 27 \mathrm{sec}$, $50 \mathrm{sec}, 1 \mathrm{~min} 30 \mathrm{sec}, 2 \mathrm{~min} 45 \mathrm{sec}, 5 \mathrm{~min}, 7 \mathrm{~min} 30 \mathrm{sec}, 11 \mathrm{~min}$ $15 \mathrm{sec}, 16 \mathrm{~min} 52 \mathrm{sec}$, and $25 \mathrm{~min}$. Two additional inspection durations, namely $1 \mathrm{~h}$ and $21 / 2 \mathrm{~h}$ were added for two of the six Os. Several days were allowed to elapse, after any of the longer intervals of inspection, until the $\mathrm{O}$ was asked to inspect again. Another precaution that was taken to minimize cumulative effects during the experiment was to alternate the pairings of color with pattern. That is, if green horizontal lines and magenta vertical lines had been used in the first inspection, magenta horizontals and green verticals were used in the next. The order of inspection intervals was not randomized, but was based on the availability of time in terms of the Os' daily and weekly schedules. For no O, however, was there an orderly progression of inspection times from short to long, or vice versa.

\section{Routine of Inspection and Testing}

Eacl, session of the experiment began with a preliminary series of settings by the $O$ of the rotatable disk. Before each setting, the $E$ displaced the disk by a variable amount, and the $O$ turned the disk by hand to achieve a color match between the horizontal and vertical gratings of the test field.

The $O$ made five separate settings of the disk. Then the first test slide was removed, and the second one, oppositely polarized, was used for five more settings. The median scale reading was found for the five settings for each pattern, and the separate colorimetric purity values were computed, arbitrarily giving a positive sign to scores on the green side of achromatic and a negative sign to scores on the magenta sije with reference to the vertical quadrants of the test pattern. The two purity values were then averaged for the final designation of the color match in the absence of any aftereffects. This was done prior to every inspection of colored test patterns. A grand mean of all these mean colorimetric purity values was found for each separate $O$ and was used as the reference value against which to judge all colorimetric purity settings made by that $O$ subsequent to inspection of the colored patterns. In other words, the grand mean was taken to represent each O's point of subjective equality of the colors existing prior to inspection in vertical and horizontal line patterns. On any day in which a residual aftereffect was still found to be present, the amount of the aftereffect was recorded and the $O$ was asked to wait at least 1 more day before proceeding to another interval of inspection.

After completion of the 10 preinspection test settings, the test apparatus was switched off and the inspection apparatus switched on for a predetermined duration. The $O$ was instructed to fixate steadily the center of the inspection field as the two differently colored patterns of horizontal and vertical lines were presented in alternation. At the moment that the inspection was completed, a timer was started and the testing field was turned on. The $O$ then made five settings of the rotatable disk with each of the two oppositely polarized test patterns, as in the preinspection procedure described above. About $2 \mathrm{~min}$ were required for the 10 settings. The median settings were recorded with reference to the time at the middle of that interval, as indicated on the timer. Settings were repeated at such intervals of time (minutes) after inspection as would permit the graphing of the time course of the decaying aftereffects as shown in Fig. 2. Strong aftereffects could not be measured initially, as explained above, because of the limitations of the testing equipment. Thus, the $O$ simply waited until color matches could be made, and then continued to make settings at roughly equal logarithmic intervals of time until full decay of the aftereffects had been noted.

\section{RESULTS}

Figure 2 presents sample curves for the strengths of aftereffect measured at various times during the process of decay that follows various intervals of inspection. The three sets of curves are for Os showing strong, intermediate, and weak aftereffects of color. Because of the consistent individual differences, it is not appropriate to average the results over all six Os. Instead, we have chosen to display sample curves for three individual Os, one from each of the three pairs. The full array of results on all Os is far too extensive for inclusion in this article, but it is available on request from the authors. A total of 64 such curves were obtained throughout the experiments. The ordinate is a linear scale of the change in colorimetric purity that was needed to cancel the aftereffects. Each data point represents the average colorimetric purity value at the time indicated on the abscissa.

It is evident from these curves that, while the colorimetric purities are never large, they exhibit a systematic relationship to duration of the preceding interval of inspection. They decline monotonically with time after inspection, rapidly at first and then more slowly out to a limit of a few hours or days. Individual Os show similar shapes of curve, but differ 

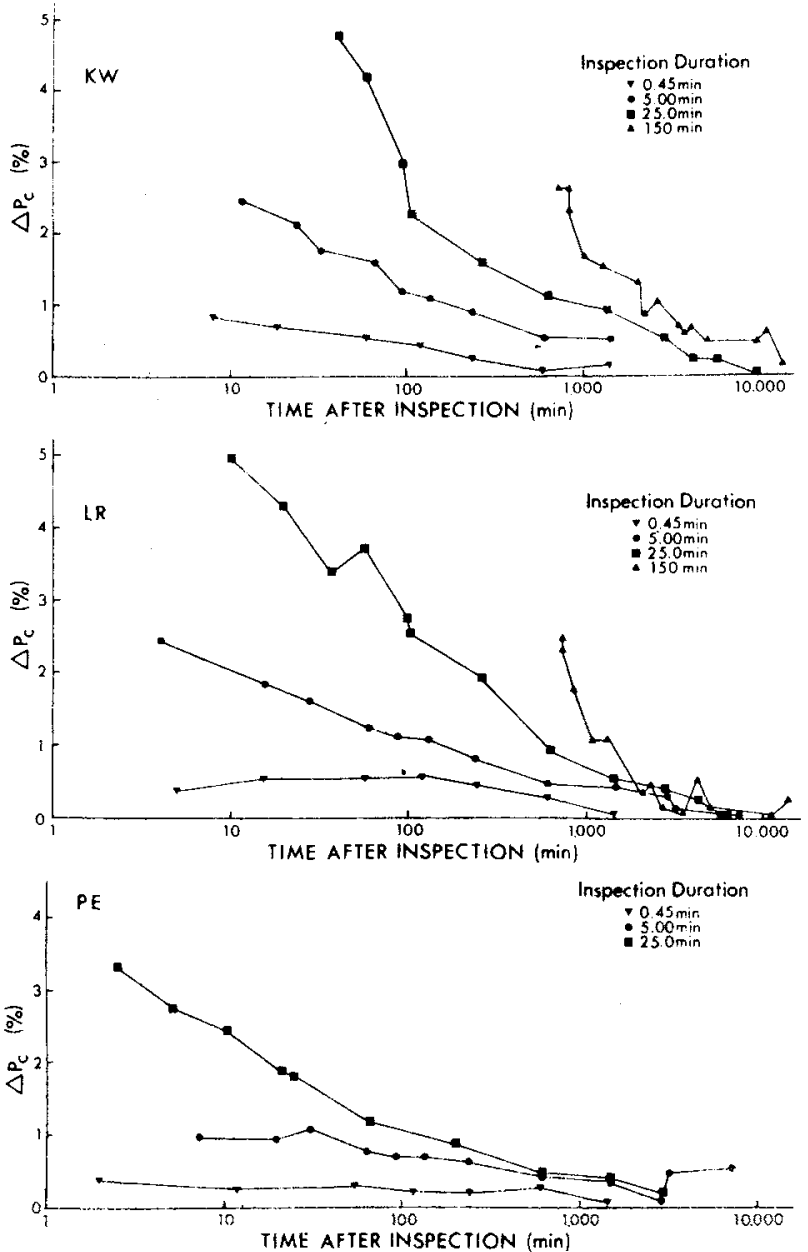

Fig. 2. Sample results for three Os on decay of aftereffect after various durations of inspection. On the logarithmic scale of decay times, 1 day is $1,440 \mathrm{~min}, 1$ week is $10,080 \mathrm{~min}$.

in the absolute amounts of the effect. Some random variability is evident, especially for settings near zero, but it is clear that the majority of the curves are nearly linear in this semilogarithmic plot. Curves for relatively high degrees of aftereffect are negatively accelerated, however, and would be almost as well described in their middle sections by a double logarithmic fit. Such a fit does not succeed, however, in representing the data at either the high or the low extremes of ordinate values. Hence, we prefer at this time not to speculate on complex forms of equation that will handle all the data, and we have instead made a separate empirical fit of the expression $\Delta \mathrm{P}_{\mathrm{c}}=$ $\mathrm{a}-\mathrm{b} \ln \mathrm{t}$ for each of the 64 decay functions, samples of which appear in Fig. 2. In this expression, $\Delta \mathbf{P}_{\mathbf{c}}$ is our index of the aftereffect in percentage units of change in colorimetric purity; $a$ is the value of $\Delta \mathbf{P}_{c}$ at $t=1 \mathrm{~min} ; \mathrm{b}$ is a slope constant indicating the rate of decay; and $t$ is time, in minutes, after inspection.

Figure 3 describes the course of establishment of the aftereffects of color. Ordinate values are the same as in Fig. 2, but on the abscissa is a logarithmic scale of the duration of inspection in minutes. Plotted points are not individual data; they are derived values based on interpolation from original curves, such as those plotted in Fig. 2. Each curve shows the increasing amount of aftereffect built up by successively longer durations of inspection, covering the range from $0.25 \mathrm{~min}(15 \mathrm{sec})$ to $150 \mathrm{~min}\left(2 \frac{1 / 2}{\mathrm{~h}}\right)$.

The increase in aftereffect is a roughly linear one when plotted against a logarithmic scale of inspection time, but there is some evidence of a positive
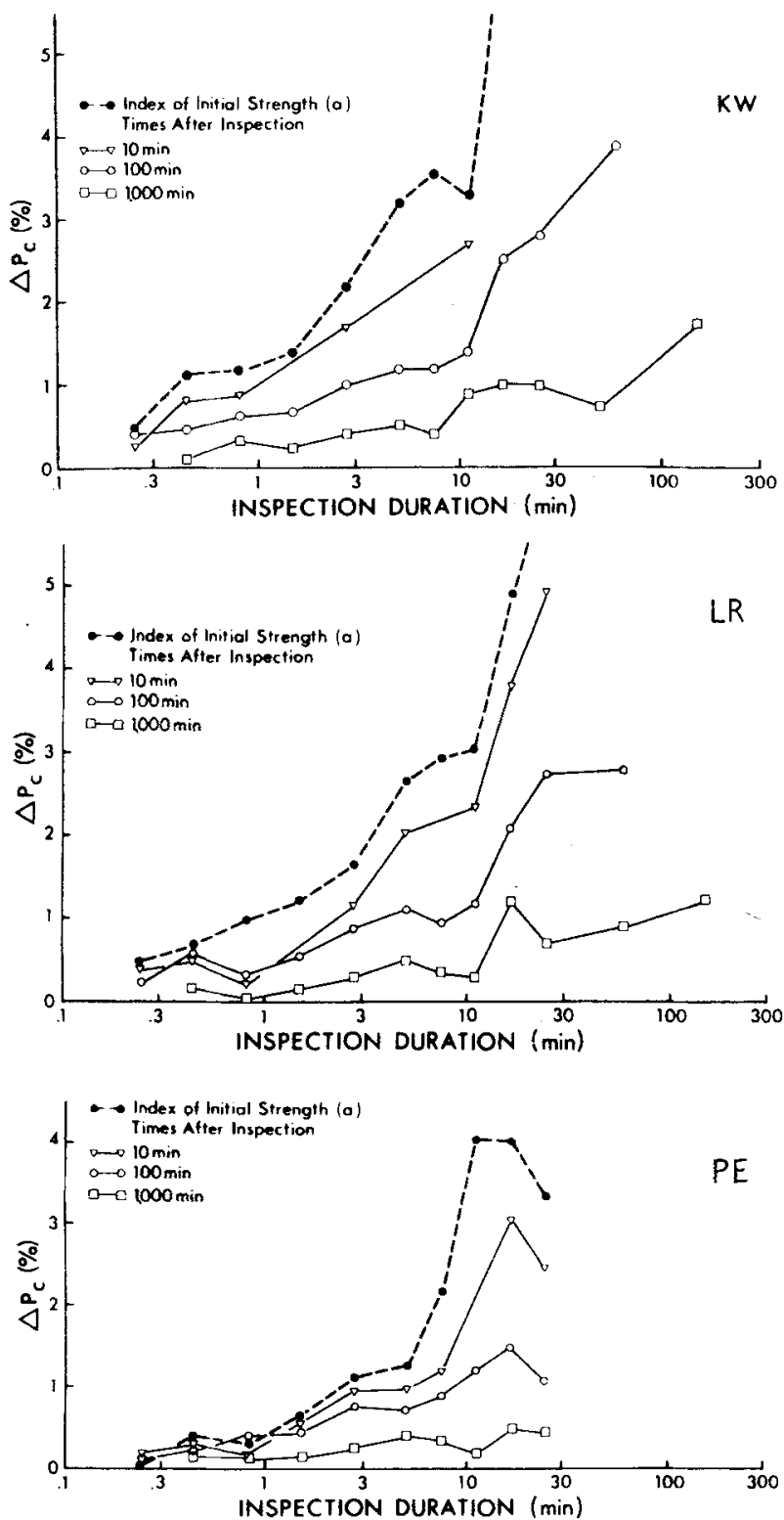

Fig. 3. Derived curves on the course of building up the aftereffect induced by alternate inspection of oppositely colored horizontal and vertical line gratings. Points are not data points, but values interpolated from decay curves, such as those shown in Fig. 2. 


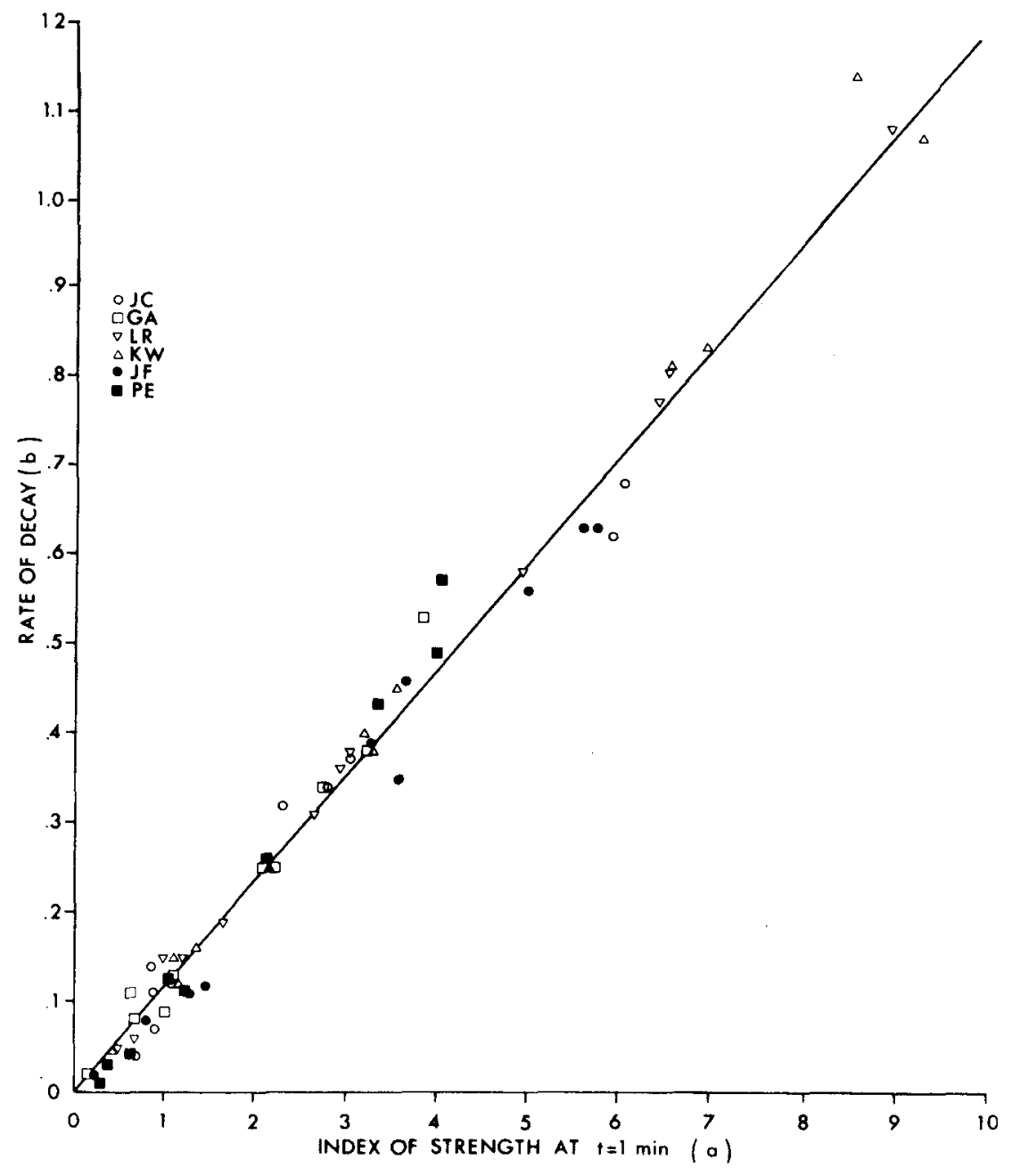

Fig. 4. Indices of rate of decay against initial strength of colored aftereffect. Each of the 64 points is derived from plotting $b$ against a obtained by fitting the equation, $\Delta P_{c}$ $=a-b \ln t$, by the method of least squares to results such as those shown in Fig. 2. This graph summarizes the data from all six Os under all conditions of the experiment. acceleration. A log-log plot gives a more nearly linear display, but shows an obvious negative acceleration for very long durations of inspection. We have made no attempt to find a more elaborate form of curve to express the data. The dashed-line curves are for values of the constant $\mathrm{a}$ in the expression $\Delta \mathrm{P}_{\mathrm{c}}=$ $a-b \ln t$ fitted by least squares to curves such as those in Fig. 2. Note that when $t=1 \mathrm{~min}, 1 \mathrm{n} t=0$, and hence $\Delta P_{c}=a$. Thus, a can be taken as an index of the initial strength of the aftereffect. Individual differences are again shown to occur, but the shapes of the curves are generally similar, if one disregards random variations.

Figure 4 is a highly condensed expression of the experimental results. It stresses the similarity, for all six Os, of the process of decay. Specifically, the ordinate is a scale of the rate of decay as given by the coefticient $\mathrm{b}$ in the empirical expression, $\Delta \mathrm{P}_{\mathrm{c}}=$ $\mathrm{a}-\mathrm{b} \ln \mathrm{t}$. The abscissa is $\mathrm{a}$, the other constant in the same equation, namely the projected value of $\Delta \mathbf{P}_{\mathrm{c}}$ at $\mathrm{t}=1 \mathrm{~min}$. Inasmuch as a can be taken as an index of the strength of the aftereffect that is built up during inspection, Fig. 4 shows that the rate of decay is highly dependent on that strength. Even though there are large individual differences in both $a$ and $b$ for any given condition of the experiment, the ratio of one to the other is nearly constant throughout.

\section{DISCUSSION}

We have obtained an empirical description of establishment and decay in a process about which little is known. The time courses we have measured are sufficiently complex to deter us from inferring from them any simple process decaying with time. On the one hand, the prolonged courses of development and decay are inconsistent with any explanation based on selective adaptation of color receptors or on the production of ordinary negative afterimages. On the other hand, explanations in terms of learning or conditioning would seem to demand that the same colors be associated with the patterns during acquisition and forgetting, whereas in all these data it is opposite colors that are seen as aftereffects of 
inspection. Any explanation, however, must also consider our finding of wide individual differences in the absolute values of the color-contingent effects. Encouragenent to seek a common physiological basis for the effects is provided, nevertheless, by the very uniform dependence shown in Fig. 4 of the rate of decay on the strength of the effect.

McCollough (1965) was aware of the new experiments at that time showing that specific cortical neurons are tuned for orientation of lines or edges (Hubel \& Wicsel, 1962). But work had barely begun on the acquisition and loss of such orientation-specific effects during normal and abnormal development or during use or disuse of vision. Reports by Wiesel and Hubel (1963) and Hirsch and Spinelli (1970) started an avalanche of studies in which kittens were deprived of vision monocularly or binocularly, totally or with partial restriction of patterns or visual fields, and the results have been evaluated by microelectrode studies of cells of the visual cortex. Still more recent are studies of the underlying histological abnormalities that may occur, depending on the animal's stage of development and the nature of the deprivation. At the same time, the behavioral deficits have been widely explored, and this work has even been extended to include the results of high astigmatism on human meridional deficits in acuity. [See recent accounts by Berkley (1970), Henry \& Bishop (1971), Blakemore (1973), Freeman, Mitchell, \& Millodot (1972), Pettigrew, Olson, \& Hirsch (1973), Sherman (1973), and Spinelli, Hirsch, Phelps, \& Metzler (1972)].

The above findings suggest that we inquire as to whether our Os normally have a perfect color balance among the cells tuned for horizontal lines, for example, as compared to those tuned for vertical. It is conceivable that people who happen to spend the formative years of their childhood in a house with green vertical striped wallpaper, for example, might thereby have had their color equilibrium permanently shifted toward the magenta side for vertical perception. One of our six Os does, indeed, consistently make preinspection settings that are different by about $0.70 \%$ on a colorimetric purity scale, indicating that the vertical line gratings are consistently seen as more magenta than horizontal ones. The corresponding settings for the other five Os range from $0.28 \%$ in the same direction to $0.18 \%$ in the opposite direction. While all these values are small, two of the six are significantly different from zero. We are left with the conclusion that, while most people see horizontal and vertical lines as having the same color, a sufticiently sensitive test of the $\mathrm{McC}$ ollough effect reveals some systematic individual differences. Inasmuch as these differences are pattern-contingent, they cannot be the result of color anomalies. Hence, they must reflect genetic or acquired imbalances for color among the populations of cortical cells tuned for separate orientations.
McCollough (1965) drew attention to the fact that prolonged adaptation to the wearing of prisms has many effects similar to those in her own experiment. The aftereffects are opposite in color from the original bands that appear along edges during the wearing of the prisms (Kohler, 1964). The magnitude and time course of both the original perceptual adaptation and the aftereffects after removal of the prisms are related to the contrast that is present in the original patterns (Hay, Pick, \& Rosser, 1963). Furthermore, the fringe effects are not transferable from one eye to the other (Hajos \& Ritter, 1965). Hence, it was McCollough's contention that these color fringe effects, like those of her own experiment, might be explained in terms of "color adaptation of oppositely oriented, vertical edge detector systems."

Due consideration must also be given to the recent blossoming of experiments on channels for the analysis of orientation and spatial frequency in the visual system (see Campbell \& Robson, 1968; Westheimer, 1972; Sekuler, 1974). These have emphasized the application of Fourier analytic techniques to the study of pattern vision. Specifically, they have shown that parallel-line gratings affect more or less independent channels of the visual system depending on their spatial frequency and orientation. Prolonged exposure to a particular grating results in a selective loss of sensitivity as shown by a raised contrast threshold. Moreover, contrast thresholds for gratings are especially low in the most favorable range (about 3 cycles $/ \mathrm{deg}$ ) of spatial frequencies. Thus, it is tempting to recast the formulation of the McCollough effect in terms of the effectiveness of grating patterns rather than merely single edges or lines; and it has already been shown (Teft \& Clark, 1968; Stromeyer, 1972) that aftereffects of color can arise on the basis of channels for spatial frequency. However, we should bear in mind that single edges can manifest the aftereffects of color, both in the McCollough effect and in the experiments on color fringes due to prisms; and we should also recognize that such features as direction of motion (Hepler, 1968), curvature (Riggs, 1973). and angles (White \& Riggs, 1974) are also effective producers of color-contingent aftereffects.

We may ask why the McCollough effect never becomes very vivid by comparison with the colors that are inspected, or with true negative afterimages. Perhaps the answer lies in the fact that the effect would not exist at all if it were not for a neural linkage of units responding selectively to features of pattern and color. Consequently, it would be expected that only a minor fraction of all the possible cells for processing visual information would take part in any particular pattern-contingent aftereffects of color. We may speculate, on the basis of studies of masking and other evidences of tuning, that the maximum band width of any given orientational channel is about 30 deg (Campbell \& Robson, 1968). This means that 
the McCollough effect, as usually tested, depends on the color-differentiating responses of no more than one-third of the total number of orientational cells, namely those tuned for angles within $15 \mathrm{deg}$ of the horizontal or vertical. We must also recognize that some color-tuned cells may not be orientationally tuned, that many orientational cells may not be color-tuned, and that many cells that are color- and orientation-tuned may not be appropriately tuned for the particular colors, orientations, spatial frequencies, or sizes of field that happen to be present. Thus, not surprisingly, no matter how long the inspection is continued or how early the aftereffects are tested, they never attain the vividness of colors evoked by stimuli having a high degree of colorimetric purity.

In consideration of all these new developments, we may now ask whether the Os in the present experiments have cortical cells that are selectively tuned for color and for such spatial features as line orientation and spatial frequency. If so, does the selectivity first appear on the particular cortical cells that may be assumed responsible for the McCollough effect, or is there a sequential analysis first by color-discriminating cells and later by cells tuned for orientation, or vice versa? The answer to this question is not presently known, but clues are beginning to come in. A two-stage or more complex sequential process would be consistent with our experimental finding that no simple monotonic function describes the courses of establishment and decay of the contingent aftereffects of color. Also consistent with a sequential model is the fact that the McCollough effect does not exhibit binocular transfer, as do many other forms of perceptual learning involving spatial, but not chromatic, processing. This may indicate that the color component is the one that fails to transfer, and that color-specific channels from the LGN are separately feeding into orientation-specific cortical cells.

All the above speculations leave us still in doubt about the fundamental nature of the effects we have measured. Our hope is that by furnishing quantitative data on the establishment and decay of the McCollough effect we have prepared the way for comparisons with other forms of perceptual adaptation and for neurophysiological studies that may ultimately reveal the locations of linkages between line orientation and color.

\section{REFERENCES}

BERKLEY, M. Visual discriminations in the cat. In W. C. Stebbins (Ed.), Animal psychophysics. New York: Appleton-CenturyCrofts, 1970.

BLAKEMORE, C. Environmental constraints on development in the visual system. In R. A. Hinde and J. Stevenson-Hinde (Eds.), Constraints on learning. London: Academic Press, 1973.

Campbell, F. W.. \& Robson, J. G. Application of Fourier analysis to the visibility of gratings. Journal of Physiology, 1968. $197,553-568$.
Exmas, P. D. . Cooper, W. E., \& Corbit, J. D. Some properties ot linguistic feature detectors. Perception \& Psychophysics, 1973. 13. $247-252$

Epstein. W. Varicties of perceptual learning. New York: McGrawHill, 1967.

Freeman. R. D.. Mitchell, D. E., \& Millodot, M. A neural effect of partial visual deprivation in humans. Science, 1972, 175, 1384-1386.

HARRIS. C. S, Perceptual adaptation to inverted, reversed, and displaced vision. Psychological Review, 1965, 72, 419.444.

hajos, A., \& Ritter, M. Experiments to the problem of interocular transfer Acta Psychologica, 1965, 24, 81-90.

HaY. J. C., PIck, H. L., \& Rosser, E. Adaptation to chromatic aberration by the human visual system. Science, 1963, 141. 167-169.

HenRy, G. H., \& Bishop, P. O. Simple cells of the striate cortex. In W. D. Neff (Ed.), Contributions to sensory physiology. New York: Academic Press, 1971.

HEPLER, N. Color: A motion-contingent aftereffect. Science, 1968. 162, 376-377.

Hirsch, H. V. B., \& Spinelli, D. N. Visual experience modifies distribution of horizontally and vertically oriented receptive fields in cats. Science, 1970, 168, 869-871.

HochberG, J. Perception, I. Color and shape, II. Space and movement. In J. W. Kling and L. A. Riggs (Eds.), Woodiorth and Schlosherg's Experimental psychology. (3rd ed.) New York: Holt, Rinchart \& Winston, 1971. Pp. 395-550.

Hubel, D. H., \& Wiesel, T. N. Receptive fields, binocular interaction and functional architecture in the cat's visual cortex. Journal of Physiology, 1962, 160, 106-154.

KOHLER, I. The formation and transformation of the visual world. Psychological Issues, 1964, 3, 1-173.

$\mathrm{McCol}$ ough, C. Color adaptation of edge-detectors in the human visual system. Science, 1965, 149, 1115-1116.

Murch, G. M. Visual and auditory perception. New York: Bobbs-Merrill, 1973.

Pettigrew, J. D., Olson, C., \& Hirsch, H. V. B. Cortical effect of selective visual experience: Degeneration or reorganization? Brain Research, 1973, 51, 345-351.

Riggs, L. A. Curvature as a feature of pattern vision. Science, 1973, 181, 1070-1072.

Rock, I. The nature of perceptual adaptation. New York: Basic Books, 1966.

SEKULER, R. Spatial vision. Annual Reviews of Psychology, 1974, 25, 195-232.

Sherman, S, M. Visual field defects in monocularly and binocularly deprived cats. Brain Research, 1973, 49, 25-49.

Spinelli, D. N., Hirsch, H. V. B., Phelps, R. W., \& Metzler, $J$. Visual experience as a determinant of the response characteristics of cortical receptive fields in cats. Experimental Brain Research, 1972, 15, 289-304.

Stromeyer, C. F., III. Edge-contingent color aftereffects: Spatial frequency specificity. Vision Research, 1972. 12. 717-734.

Stromeyer, C. F., III., Lange, A. F., \& Ganz, L. Spatial frequenicy phase effects in human vision. Vision Research, 1973 , 13. 2345-2360.

TEFT, L., \& C CARK, F. The effects of stimulus density on orientation specific aftereffects of color adaptation. Psychonomic Science, 1968, 11, 265-266.

Westhermer, G. Visual acuity and spatial and modulation thresholds. In D. Jameson and L. M. Hurvich (Eds.), Visual psychophysics. Vol. V11/4. Handbook of sensory physiology. Berlin: Springer, 1972.

White, K. D., \& Riggs, L. A. Angle-contingent color aftereffects. Vision Research, 1974, in press.

Wiesel. T. N., \& Hubel, D. H. Single-cell responses in striate cortex of kittens deprived of vision in one eye. Journal of Neurophysiologv, 1963, 26, 1003-1017. 


\section{NOTE}

1. The total luminance of the bright stripes of the test pattern remained nearly constant at $6.8 \mathrm{fL}$. This was made up of $5.0 \mathrm{fL}$ of the magenta, green, and white lights contributed by the color-mixing projector plus $1.8 \mathrm{fL}$ of ambient light contributed by the flourescent fixture. Our dependent variable was a calculated ratio, detailed below, of luminance contributions from each color and from the white lights, and was very nearly the same as colorimetric purity $\left(\mathrm{P}_{\mathrm{c}}\right)$. Strictly speaking, our use of $\mathbf{P}_{\mathrm{c}}$ could only be justified if the dominant wavelengths (including the extra-spectral point assigned to magenta) of the color filters were truly complementary, and monochromatic, i.e., of $100 \%$ colorimetric purity. Inasmuch as our dominant wavelengths were not precisely complementary and our colors not quite monochromatic, we estimate that the values derived from our luminance measurements should be corrected by a factor of 0.88 to yield values of $P_{c}$ as conventionally defined, namely $P_{c}=$ $L_{\lambda} /\left(L_{\lambda}+L_{W}\right)$, where $L_{\lambda}$ is the luminance of the component of monochromatic light of dominant wavelength, $\lambda$, and $L_{W}$ is the luminance of white component. This makes only a trivial change, well within the range of experimental error. Our working formula is $P_{c}=\left\|\left[.88\left(L_{M}-L_{G}\right)\right] /\left[L_{M}+L_{G}+L_{W}+L_{B}\right]\right\|$, where $P_{c}$ is the colorimetric purity and the luminance values, $L$, represent the components due to the magenta and green filters and to the light from the white beam of the projector and the ambient background illumination.

(Received for publication April 24, 1974; accepted June 21, 1974.) 